\title{
Comparison of efficacy and visual quality after CWFG-FS-LASIK, AF-FS-LASIK and SMILE for correcting myopia with asymmetry mid-to-high astigmatism
}

zhongxiu zhao

Aier Eye Hospital Group https://orcid.org/0000-0001-8985-849X

Miaomiao Zhu

Aier Eye Hospital Group

Qian Jian

Aier Eye Hospital Group

Xialu Liu

aier

Pingting $\mathrm{Ma}$

Aier Eye Hospital Group

Ping Li

Aier Eye Hospital Group

Yanli Peng ( $\triangle$ pengyanli@aierchina.com )

Research article

Keywords: corneal wavefront-guided femtosecond LASIK, coma, SMILE, FS-LASIK, corneal asymmetry, astigmatism

Posted Date: May 1st, 2020

DOl: https://doi.org/10.21203/rs.3.rs-19318/v2

License: (c) (i) This work is licensed under a Creative Commons Attribution 4.0 International License. Read Full License 


\section{Abstract}

Background: Individualized ablation is not only able to correct corneal low-order aberrations but also to improve corneal high-order aberrations in patients with corneal asymmetry. In this study, we compared the effects of three different surgical methods, namely, corneal wavefront-guided femtosecond laser assisted in-situ keratomileusis (CWFG-FS-LASIK), aberration-free femtosecond laser assisted in-situ keratomileusis (AF-FS-LASIK) and small incision lenticule (SMILE), in patients with mid-to-high astigmatism asymmetric corneas (1-4D).

Methods: One hundred-fourteen eyes from 58 patients were enrolled in this retrospective study. We measured and compared the best corrected visual acuity (BCVA), uncorrected visual acuity (UCVA), residual astigmatism, total coma, vertical coma $\left(Z_{3}{ }^{-1}\right)$, horizontal coma $\left(Z_{3}{ }^{1}\right)$, modulation transfer function (MTF) and point spread function (PSF) preoperatively and three months postoperatively.

Results: The visual acuity of patients in the three groups was increased after surgery, but the improvements in visual acuity and residual astigmatism among them were not significantly different. However, the improvements in 4-mm and 6-mm total coma, $Z_{3}{ }^{-1}$ and $Z_{3}{ }^{1}$ in patients who underwent CWFG-FS-LASIK were better than those in patients who underwent AF-FS-LASIK and SMILE. Consistent with these results, the improvements in MTF and PSF ( $3 \mathrm{~mm}$ and $5 \mathrm{~mm}$ ) in the CWFG-FS-LASIK group were better than those in the AF-FS-LASIK and SMILE groups.

Conclusions: Among surgeries for treating patients with mid-to-high astigmatism in asymmetric corneas, the selective coma-guided modality was able to decrease the coma of original cornea, improve visual acuity and optimize the visual quality of patients.

\section{Background}

Excimer laser refractive surgery is an effective technique for the correction of 2nd-order myopic, hyperopic, and astigmatic refractive errors. Femtosecond laser assisted in-situ keratomileusis (FS-LASIK) has become a staple among the refractive surgeries because of its predictable, effective, and well tolerated ${ }^{[1]}$. Small incision lenticule extraction (SMILE), as a novel developed corneal refractive technique with only a small incision required, which is less invasive than FS-LASIK. While, a fixed surgical method may not be suitable for all patients with ametropia, especially for patients with mid-to-high astigmatism in asymmetric corneas, which is usually accompanied by increased corneal aberrations. Higher order aberrations (HOAs) are one of the main factors that affect postoperative visual quality after refractive surgery. HOAs are closely related to preoperative or intraoperative corneal aberrations and are also associated with corneal repair after surgery ${ }^{[2]}$. Currently, new algorithms had been developed to minimize the inductions of HOAs. Aberration-Free treatment is one of these new algorithms which provided by Amaris laser platforms. In that case, aberration-free LASIK (AF-FS-LASIK) treatment could be considered as a possible method to correct both lower order aberrations and corneal asymmetry in the same time ${ }^{[3]}$. However, the asymmetric of corneal which often been noticed in patients with moderate to high 
astigmatism can not be treated by abovementioned 2 types of procedures which been considered to be related to corneal coma that might cause vision quality problems like glare.

Therefore, an individualized ablation that is not only able to correct corneal low-order aberrations but also to improve corneal HOAs, especially coma, caused by preoperative corneal morphological asymmetry is becoming more advantageous. In this scenario, corneal wavefront guided (CWFG) treatment could be considered as a possible method to correct both lower order aberrations and corneal asymmetry in the same time. In this study, a SCHWIND AMARIS excimer laser from Germany was used, and a Keratron scout corneal topographic map guided the treatment of patients with high astigmatism by corneal wavefront-guided femtosecond femtosecond laser assisted laser in-situ keratomileusis (CWFG-FS-LASIK), AF-FS-LASIK or SMILE.

\section{Material And Method}

\section{Study Group and Design}

This retrospective observational study was performed at the Department of Ophthalmology, Chongqing Aier-Mega Eye Hospital, Aier Eye Hospital Group. Informed written consent was obtained from all patients. The inclusion criteria were as follows: age $\geq 18$ years, total coma $\geq 0.25 \mathrm{Dep}$, best corrected visual acuity (BCVA) $\leq 1.0$, Pentacam (Oculus Optikgeräte $\mathrm{GmbH}$, Wetzlar, Germany) scans of the front surface of the cornea showing a $3 \mathrm{~mm}$ height difference of refraction more than $1.25 \mathrm{D}$, and stable refraction for two years prior. The exclusion criteria were as follows: conical cornea, scarred cornea, corneal lesions, active ocular disease, or autoimmunological disease. Finally, from 2018 to 201958 patients (114 eyes) who underwent CWFG-FS-LASIK, AF-FS-LASIK or SMILE and were followed up for three months were included.

\section{Surgical Technique}

The total coma of patients was screened in natural light by a Keratron scout topographic map. We selected eight topographic map pictures and imported them into an SCHWIND AMARIS excimer laser. All operations were performed on the patient's superior side by the same surgeon. In the patients who underwent CWFG-FS-LASIK, we designed the parameters of surgery with a correcting coma before importation into a SCHWIND AMARIS excimer laser. Then, a corneal flap (thickness of $100 \mu \mathrm{m}$, diameter of $8.1 \mathrm{~mm}$ ) was made with VisuMax3.0. Finally, the corneal stromal layer was removed in a selective coma-guided mode. Compared with the patients who underwent CWFG-FS-LASIK, the patients who underwent AF-FS-LASIK did not undergo coma correction before surgery. In the patients who underwent SMILE, a 2-mm curved incision was made at the 10:30 position by VisuMax3.0, the corneal cap was 120 $\mu \mathrm{m}$, and the diameter of the lens was 6.3 to $6.5 \mathrm{~mm}$ or was slightly adjusted. All patients were administered levofloxacin drops 4 times a day for one week, loteprednol etabonate ophthalmic suspension 4 times a day for four weeks, and sodium hyaluronate drops 4 times a day for three months.

\section{Preoperative and Postoperative Examinations}


All patients underwent a general ophthalmologic examination preoperatively: visual acuity, BCVA, refraction, tonometry, slit-lamp evaluation, topography specular microscopy, and fundoscopy were assessed. Three months postoperatively, uncorrected visual acuity (UCVA), residual astigmatism, coma (total, vertical and horizontal), modulation transfer function (MTF), point spread function (PSF) were measured.

\section{Statistical Analysis}

All statistical analyses were performed by SPSS (version 19; SPSS, Inc.). Figures were drawn with GraphPad Prism (version 8; GraphPad Software). Data are presented as the mean \pm standard deviation. Student's t-test was used for normally distributed data, and the Mann-Whitney test was used for nonnormally distributed data. One-way analysis of variance (ANOVA) was used to analyze the difference among the three groups with different operative methods, and LSD was used to perform additional multiple comparison tests. $P<0.05$ was considered statistically significant.

\section{Results}

This study finally included 114 eyes (58 patients): 39 eyes (20 patients) in the CWFG-FS-LASIK group; 38 eyes (19 patients) in the AF-FS-LASIK group; and 37 eyes (19 patients) in the SMILE group. The demographics of the study participants and the parameters of the eyes included in each group are summarized. None of the patients had an infection or a transposition, fold, or decentered ablation of the supracorneal flap. The general situation of the patients before surgery is shown in Table 1.

\section{Visual acuity}

The BCVA of the CWFG-FS-LASIK group, AF-FS-LASIK group and SMILE group was $0.0077 \pm 0.027$, $0.0105 \pm 0.031$ and $0.0135 \pm 0.035$, respectively, with no significant difference $(P>0.05)$. The UCVA of the CWFG-FS-LASIK group, AF-FS-LASIK group and SMILE group was $0.3641 \pm 0.341,0.3658 \pm 0.345$ and $0.4351 \pm 0.344$, respectively, 3 months after the operation, with no significant difference $(P>0.05)$. The postoperative UCVA of patients in three groups were all increased compared with the preoperative BCVA, but the magnitude of the increase among them was no different (Figure 1).

\section{Residual astigmatism}

The residual astigmatism in the CWFG-FS-LASIK group, AF-FS-LASIK group and SMILE group was $-0.43 \pm 0.14,-0.44 \pm 0.16$, and $-0.43 \pm 0.16$, respectively. There was no significant difference among the three groups $(P>0.05)$ (Figure 2).

\section{Change in coma aberration}

We compared the total coma aberration, $Z_{3}{ }^{-1}$ and $Z_{3}{ }^{1}$ in the three groups three months after surgery. The total coma aberration ( $4 \mathrm{~mm}$ and $6 \mathrm{~mm}$ ) in the CWFG-FS-LASIK group decreased compared with preoperative data, while it increased in the AF-FS-LASIK group and the SMILE group. The improvement in 
the total coma aberration in the CWFG- FS-LASIK was better than that in the other groups (Figure 3A). The 4-mm $\mathrm{Z}_{3}{ }^{-1}$ and 6-mm $\mathrm{Z}_{3}{ }^{-1}$ in the CWFG-FS-LASIK group decreased after surgery; on the contrary, it increased in the AF-FS-LASIK group and the SMILE group. The improvement in $\mathrm{Z}_{3}^{-1}$ in the CWFG- FSLASIK group was better than that in the other two groups (Figure 3B). Finally, we compared pre-and postoperative 4-mm $Z_{3}{ }^{1}$ and 6-mm $Z_{3}{ }^{1}$, which was significantly decreased in the CWFG-FS-LASIK group but increased in the AF-FS-LASIK group and the SMILE group. The improvement in 4-mm $Z_{3}{ }^{1}$ was better than in the other two groups, and there was no significant difference in $6-\mathrm{mm}_{3}{ }_{3}{ }^{1}$ (Figure $3 \mathrm{C}$ ). Pre-and postoperative data are shown in Table $2 \mathrm{~A}$.

\section{Changes in the MTF and PSF}

The MTF and PSF were measured by I-Trace (Tracey Technologies, Houston, TX). The MTF ( $3 \mathrm{~mm}$ and 5 $\mathrm{mm}$ ) in the CWFG- FS-LASIK and SMILE groups increased after 3 months but decreased in the AF-FSLASIK group. The improvements in the MTF $(3 \mathrm{~mm}$ and $5 \mathrm{~mm}$ ) in both the CWFG-FS-LASIK and SMILE groups were superior to those in the AF-FS-LASIK group (Figure 4A). Regarding the PSF ( $3 \mathrm{~mm}$ and $5 \mathrm{~mm}$ ) of three groups, the PSF in the CWFG-FS-LASIK and SMILE groups were increased but decreased in the AF-FS-LASIK group. The improvements in the PSF $(3 \mathrm{~mm}$ and $5 \mathrm{~mm})$ in the CWFG-FS-LASIK and SMILE groups were superior to those in the AF-FS-LASIK (Figure 4B), similar to the MTF parameter. Pre-and postoperative data are shown in Table 2.

\section{Discussion}

Astigmatism is a type of refractive error in which the eye does not focus light evenly on the retina and is unable to form a clear image. Corneal astigmatism can be divided into a round shape, an oval shape, an asymmetric bow-tie shape, and an irregular shape according to the corneal shape; among these shapes, the most common is an asymmetrical bow-tie shape, accounting for approximately $50.9 \%$ of cases ${ }^{[3]}$. Traditional corneal refractive surgery has a better effect for correcting low-order aberrations (myopia, astigmatism), but additional HOAs often develop after surgery, or the original HOAs increase, which are considered a cause of decline in postoperative visual quality ${ }^{[3,5]}$. Postoperative HOAs are mainly derived from the following causes: corneal flap production and its ablation depth, namely, the deeper the ablation, the more HOAs that are introduced; cooperation from the patient and eye position during surgery; and corneal wound healing, corneal morphology and biomechanical changes after surgery. These factors will also cause additional aberrations. From clinical observations, in some obvious cases of corneal astigmatism with an asymmetric bow-tie shape, they are often accompanied by HOAs, especially coma [68]. Visual quality is affected by coma aberrations, which make patients more prone to nighttime visual quality, such as halo, glare, difficulty in near vision, smearing, and poor dark vision ${ }^{[9]}$. Therefore, for such patients who are admitted after undergoing a traditional surgical method for correcting corneal refraction, the original asymmetry of the cornea may be aggravated, and an increase in the original HOAs may cause a decrease in visual quality after surgery ${ }^{[10]}$. In this study, patients with myopia and asymmetric mid-to-high astigmatism mainly accompanied by increased coma underwent individual treatments. The 
most commonly used personalized surgery is corneal topographical guidance and wavefront aberration guidance. The purpose of corneal topographical map guidance is to reduce corneal irregularity and thus reduce high-priced aberrations. Corneal topography should be the first choice to guide surgery for irregular astigmatism of the cornea. Wavefront aberration guidance is based on full-eye aberrations and is affected by intraocular astigmatism, such as the tear film, pupil, adjustment, and lens ${ }^{[11]}$. The operative mode guided by wavefront aberration is to directly reduce high-order aberration as the ultimate goal. It can not only improve the regularity of the anterior surface of the cornea but also make the morphology of the posterior surface of the cornea more regular. Astigmatism of the asymmetric cornea is mainly due to the increase in coma. According to its direction, it is divided into $Z_{3}{ }^{-1}$ and $Z_{3}{ }^{1} ; Z_{3}{ }^{-1}$ mainly affects the visual quality along the vertical meridian. Most of the patients we selected had vertically asymmetrical astigmatism. The increase in $\mathrm{Z}_{3}{ }^{-1}$ was also the main factor before surgery. Therefore, this study adopted the unique selective coma elimination mode with the SCHWIND AMARIS excimer laser, which is more targeted. $Z_{3}{ }^{-1}$ was eliminated, and $Z_{3}{ }^{1}$ was improved to some extent but not as much as $Z_{3}{ }^{-1}$. Fernández-Sánchez $V$ et al ${ }^{[12]}$ found that the visual quality of patients was improved after greater aberration was corrected. We found that the total coma in the CWFG-FS-LASIK group at 3 months after surgery was reduced compared with that before surgery but was increased in the AF-FS-LASIK and SIMLE groups. This may be because FS-LASIK is affected by flapping, coordination, tracking, and biomechanics; thus, it is more easy to introduce additional HOAs. However, SMILE has less factors of interference than traditional LASIK surgery due to the lensless mode ${ }^{[13,14]}$; furthermore, SMILE causes less HOAs ${ }^{[15,16]}$. The results also showed that SMILE group had less introduction to coma of a surgical origin than the AF-FSLASIK group. In this study, there was no significant difference in total coma between the AF-FS-LASIK and SMILE groups. On vector analysis of coma, the CWFG-FS-LASIK group had better improvement in the vertical coma, while the other groups had no significant improvement and even introduced a surgical source of $Z_{3}{ }^{-1}$. Furthermore, CWFG-FS-LASIK was able to correct part $Z_{3}{ }^{1}$, while AF-FS-LASIK and SMILE were not. This finding may be related to the small sample size in this study and the introduction of a surgical source.

We also observed the correction of low-order aberrations in the three groups for mid-to-high asymmetry astigmatism. The visual acuity of patients in the three groups after 3 months was improved compared with the BCVA before the surgeries, but there was no significant difference among the groups. We used residual astigmatism to evaluate the correction of astigmatism and found that the residual astigmatism in CWFG-FS-LASIK group was low; however, there was no significant difference among the groups. These results showed that although CWFG-FS-LASIK was able to improve coma better than other two surgical methods, but it had no obvious advantage in improving vision and residual astigmatism. Therefore, was the surgical treatment effect in the three groups the same? Visual acuity and diopter, as low-order aberrations, are primary indicators for evaluating visual quality ${ }^{[17,18]}$. Therefore, to further compare the visual quality among the three groups, we selected the i-Tace inspection equipment to observe the MTF and PSF to further observe the impact of the three methods on visual quality. MTF describes the relationship between object image contrast and optical system imaging quality at different spatial 
frequencies, is not affected by subjective factors, and objectively reflects the optical imaging quality of the entire refractive system of the eyeball. The larger the MTF value is, the clearer the imaging, and the better the visual quality ${ }^{[19]}$. The PSF is another an important objective indicator to measure the quality of retinal imaging. The image quality was evaluated by the size of the spot area and the intensity of the spot. In this study, we found that the visual quality in the CWFG-FS-LASIK and SMILE groups improved both during the day and at night compared with before surgery, but the visual quality decreased in the AFFS-LASIK group.

\section{Conclusions}

In conclusion, CWFG-FS-LASIK was superior to decrease coma and improve visual quality in patients with myopia with mid-to-high astigmatism. However, this study focused on HOAs of the cornea, especially the coma. There are limitations of this study in that no attention was paid to the changing factors of intraocular aberrations. This study mainly discussed the analysis of relatively stable 3-month data; however, a longer time for research is needed. At present, in addition to aberration-guided surgical methods, topographic map-guided surgical methods are also used in personalized treatment schemes. Due to the limitations of the equipment, this study did not include corneal topographic map-guided methods for comparison, particularly to evaluate corneal asymmetry compared with AF-FS-LASIK and SMILE. Longer-term and larger studies will be needed in the future.

\section{Abbreviations}

CWFG-FS-LASIK

Selective coma guidance femtosecond Laser in-situ keratomileusis

AF-FS-LASIK

Aberration-Free femtosecond Laser in-situ keratomileusis

SMILE

Small incision lenticule;BCVA:best corrected visual acuity;

UCVA

uncorrected visual acuity; $Z_{3}{ }^{-1}$ :vertical coma; $Z_{3}{ }^{1}$ :horizontal coma;

MTF

Modulation Transfer Function; PSF:Point Spread Function;

HOAs

High-order aberrations.

\section{Declarations}

\section{Ethics approval and consent to participate}

This retrospective observational study was approved by the Ethics Committee of Chongqing Aier-Mega Eye Hospital, Aier Eye Hospital Group. Informed written consent was obtained from all patients. 


\section{Consent to publish}

Not applicable.

\section{Availability of data and materials}

The data have not been placed in any online data storage. All data generated and analyzed during this study are available upon request from the correspondence author: Yanli Peng, E-Mail: pengyanli@aierchina.com.

\section{Competing interests}

The authors declare that they do not have any conflict of interests.

\section{Funding}

None.

\section{Authors' Contributions}

ZXZ and YLP were primarily responsible for designing the study. ZXZ, QJ and MMZ performed data analysis, as well as drafting of the manuscript. XLL, PTM and PL collected data. All authors reviewed and approved the final manuscript.

\section{Acknowledgment}

We want to thank the support and help of Dr. Weili Wang (the Second Affiliated Hospital of Third Military Medical University).

\section{Author details}

${ }^{1}$ Department of Ophthalmology, Chongqing Aier-Mega Eye Hospital, Aier Eye Hospital Group, Chongqing 400060, China. ${ }^{2}$ Aier School of Ophthalmology, Central South University, Changsha 410000, Hunan Province, China

\section{References}

[1] Chen SP, Manche EE. Patient-reported vision-related quality of life after bilateral wavefront-guided laser in situ keratomileusis. J Cataract Refract Surg, 2019, Jun; 45(6): 752-759.

[2] Li L, Cheng GPM, Ng ALK et al. Influence of refractive status on the higher-order aberration pattern after small incision lenticule extraction surgery. Cornea. 2017;36(8):967-72

[3] Jin HY, Wan T, Yu XN. Corneal higher-order aberrations of the anterior surface, posterior surface, and total cornea after small incision lenticule extraction (SMILE): high myopia versus mild to moderate 
myopia. BMC Ophthalmol. 2018 Nov 12;18(1):295.

[4] Oshika T, Klyce S D, Applegate R A, et al. Comparison of corneal wavefront aberrations after photorefractive keratectomy and laser in situ keratomileusis[J]. American journal of ophthalmology, 1999, 127(1): 1-7.

[5] Moreno-Barriuso E, Lloves J M, Marcos S, et al. Ocular aberrations before and after myopic corneal refractive surgery: LASIK-induced changes measured with laser ray tracing[J].Investigative Ophthalmology \& Visual Science, 2001, 42(6): 1396-1403.

[6] Al-Zeraid F M, Osuagwu U L. Induced high-order aberrations after laser in situ keratomileusis (LASIK) performed with wavefront-guided IntraLase femtosecond laser in moderate to high astigmatism[J]. BMC ophthalmology, 2016, 16(1): 29.

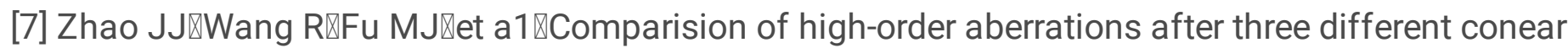
refreetive surgeries[J]『Int Eye Sci.2016,16(2):274-276》

[8] Ji PखBi HS $₫$ Wang XR.et a1 $₫$ Multiplicity of high-order aberradons changes after LASIK for myopia and

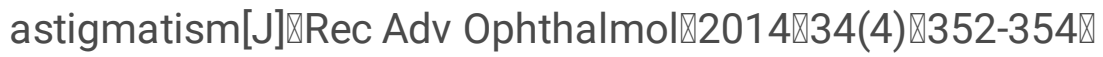

[9] Chalita M R, Chavala S, Xu M, et al. Wavefront analysis in post-LASIK eyes and its correlation with visual symptoms, refraction, and topography[J]. Ophthalmology, 2004, 111(3): 447-453.

[10] Schallhorn S C, Farjo A A, Huang D, et al. Wavefront-guided LASIK for the correction of primary myopia and astigmatism: a report by the American Academy of Ophthalmology[J]. Ophthalmology, 2008, 115(7): 1249-1261.

[11] Dick H B, Kaiser S. Dynamic aberrometry during accommodation of phakic eyes and eyes with potentially accommodative intraocular lenses[J]. Der Ophthalmologe: Zeitschrift der Deutschen Ophthalmologischen Gesellschaft, 2002, 99(11): 825-834.

[12] Fernández-Sánchez V, Ponce M E, Lara F, et al. Effect of 3rd-order aberrations on human vision[J]. Journal of Cataract \& Refractive Surgery, 2008, 34(8): 1339-1344.

[13] Pallikaris I G, Kymionis G D, Panagopoulou S I, et al. Induced optical aberrations following formation of a laser in situ keratomileusis flap[J]. Journal of Cataract \& Refractive Surgery, 2002, 28(10): 17371741.

[14] Porter J, MacRae S, Yoon G, et al. Separate effects of the microkeratome incision and laser ablation on the eye's wave aberration[J]. American journal of ophthalmology, 2003, 136(2): 327-337.

[15] Ganesh S, Gupta R. Comparison of visual and refractive outcomes following femtosecond laserassisted lasik with smile in patients with myopia or myopic astigmatism[J]. Journal of refractive surgery, 2014, 30(9): 590-596. 
[16] Lin F, Xu Y, Yang Y. Comparison of the visual results after SMILE and femtosecond laser-assisted LASIK for myopia[J]. Journal of Refractive Surgery, 2014, 30(4): 248-254.

[17] Güell J L, Pujol J, Arjona M, et al. Optical Quality Analysis System: instrument for objective clinical evaluation of ocular optical quality[J]. Journal of Cataract \& Refractive Surgery, 2004, 30(7): 1598-1599.

[18] Ondategui J C, Vilaseca M, Arjona M, et al. Optical quality after myopic photorefractive keratectomy and laser in situ keratomileusis: comparison using a double-pass system[J]. Journal of Cataract \& Refractive Surgery, 2012, 38(1): 16-27.

[19] az-Doutón F, Benito A, Pujol J, et al. Comparison of the retinal image quality with a Hartmann-Shack wavefront sensor and a double-pass instrument[J]. Investigative ophthalmology \& visual science, 2006, 47(4): 1710-1716.

\section{Tables}

\begin{tabular}{lcccc}
\hline Parameter & CWFG-LASIK & AF-FS-LASIK & SMILE & $P$ value \\
\hline Demographics & $26.06 \pm 5.98$ & $25.00 \pm 7.37$ & $25.12 \pm 5.92$ & 0.864 \\
Age (year) & 39 & 38 & 37 & \\
Number of eyes & & & $-4.58 \pm 1.18$ & 0.601 \\
Preoperative & $-4.46 \pm 1.65$ & $-4.78 \pm 0.89$ & $-2.26 \pm 0.43$ & 0.273 \\
Sphere (D) & $-1.73 \pm 0.65$ & $-1.39 \pm 0.86$ & $531.10 \pm 19.92$ & 0.843 \\
Cylinder (D) & $534.60 \pm 22.34$ & $528.00 \pm 20.54$ & $15.66 \pm 4.30$ & 0.732 \\
Corneal thickness $(\mu \mathrm{m})$ & $15.91 \pm 3.90$ & $14.47 \pm 2.35$ & \\
IOP (mmHa) & &
\end{tabular}

Table 1 Patient characteristics

IOP: Intraocular pressure

\begin{tabular}{llcccc}
\hline Parameter & Diameter & \multicolumn{3}{c}{ Post-0peration vs Pre-operation } \\
\hline \multirow{2}{*}{ Total coma aberration } & $4 \mathrm{~mm}$ & $0.17 \pm 0.07$ vs $0.29 \pm 0.04$ & $0.34 \pm 0.10$ vs $0.28 \pm 0.04$ & $0.28 \pm 0.04$ vs $0.28 \pm 0.03$ \\
& $6 \mathrm{~mm}$ & $0.22 \pm 0.12$ vs $0.34 \pm 0.08$ & $0.38 \pm 0.13$ vs $0.32 \pm 0.047$ & $0.36 \pm 0.15$ vs $0.33 \pm 0.04$ \\
Vertical coma aberration & $4 \mathrm{~mm}$ & $0.16 \pm 0.11$ vs $0.22 \pm 0.05$ & $0.26 \pm 0.13$ vs $0.21 \pm 0.05$ & $0.27 \pm 0.13$ vs $0.21 \pm 0.032$ \\
& $6 \mathrm{~mm}$ & $0.20 \pm 0.11$ vs $0.30 \pm 0.04$ & $0.38 \pm 0.10$ vs $0.30 \pm 0.05$ & $0.35 \pm 0.13$ vs $0.30 \pm 0.30$ \\
Horizontal coma berration & $4 \mathrm{~mm}$ & $0.22 \pm 0.05$ vs $0.17 \pm 0.08$ & $0.21 \pm 0.08$ vs $0.18 \pm 0.05$ & $0.21 \pm 0.07$ vs $0.18 \pm 0.03$ \\
& $6 \mathrm{~mm}$ & $0.25 \pm 0.11$ vs $0.28 \pm 0.08$ & $0.31 \pm 0.11$ vs $0.27 \pm 0.07$ & $0.32 \pm 0.13$ vs $0.27 \pm 0.04$ \\
MTF & $3 \mathrm{~mm}$ & $0.60 \pm 0.10$ vs $0.48 \pm 0.10$ & $0.42 \pm 0.10$ vs $0.48 \pm 0.09$ & $0.53 \pm 0.09$ vs $0.49 \pm 0.06$ \\
& $5 \mathrm{~mm}$ & $0.42 \pm 0.05$ vs $0.33 \pm 0.04$ & $0.31 \pm 0.06$ vs $0.35 \pm 0.04$ & $0.37 \pm 0.04$ vs $0.32 \pm 0.04$ \\
PSF & $3 \mathrm{~mm}$ & $0.53+0.10$ vs $0.40+0.13$ & $0.35+0.13$ vs $0.41+0.09$ & $0.45+0.14$ vs $0.40+0.12$ \\
& $5 \mathrm{~mm}$ & $0.43+0.08$ vs $0.33+0.07$ & $0.26 \pm 0.07$ vs $0.32+0.08$ & $0.37+0.05$ vs $0.33+0.07$ \\
\hline
\end{tabular}

Table 2 Pre- and postoperative coma, MTF and PSF data from the three surgical methods. MTF: modulation transfer function, PSF: point spread function

\section{Figures}




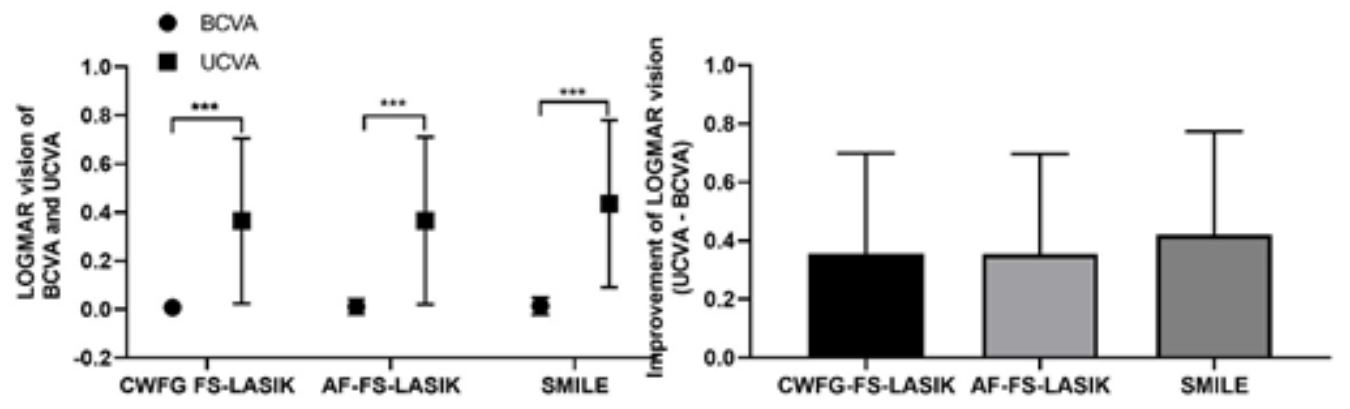

\section{Figure 1}

Comparison of the visual acuity improvement before and after the different surgical methods and the visual acuity improvement among the three groups.

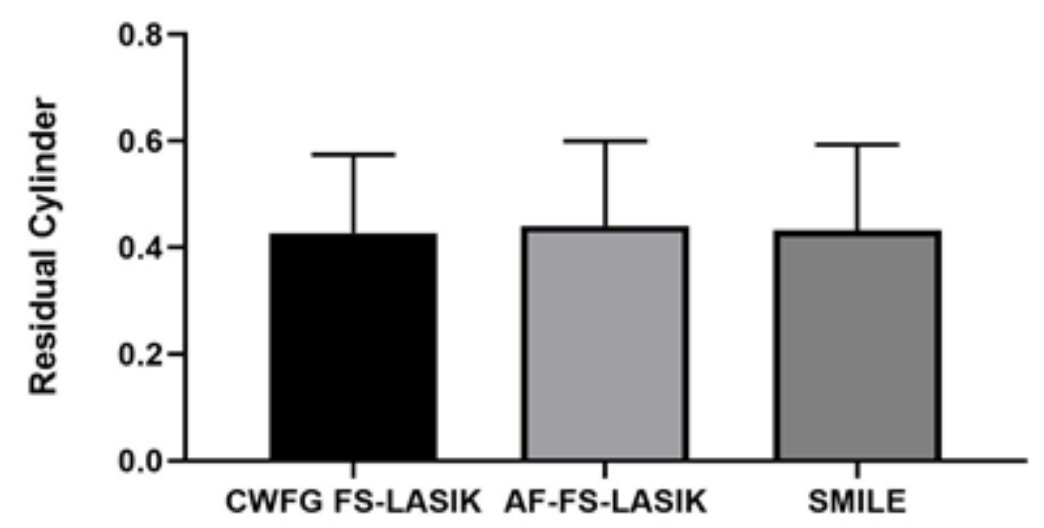

Figure 2

Residual astigmatism among the three surgical methods. 

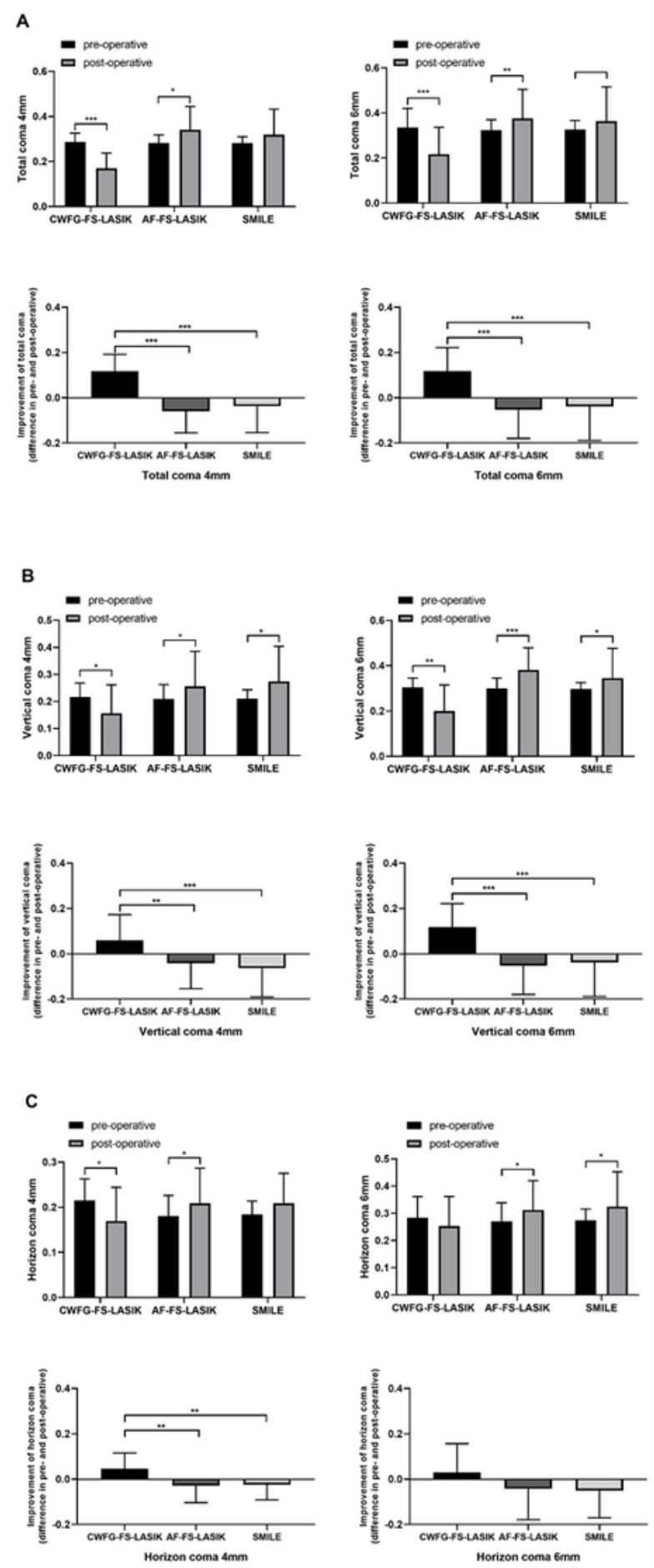

Figure 3

A Pre- and postoperative total coma and its improvement with the three surgical methods. B Pre- and postoperative vertical coma and its improvement with the three surgical methods. C Pre- and postoperative horizontal coma and its improvement with the three surgical methods. 
A
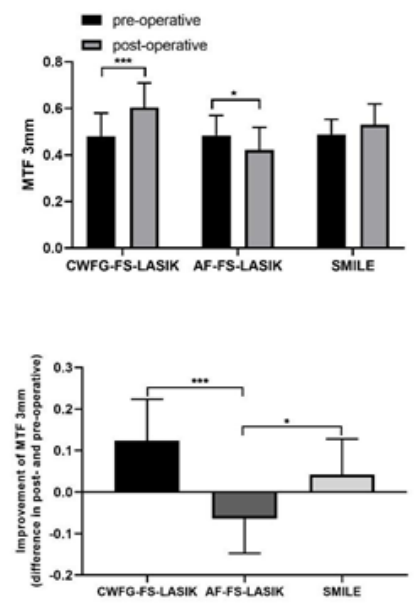

- pre-operative

-
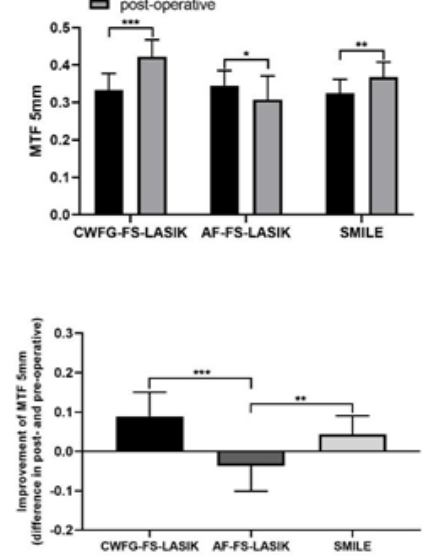
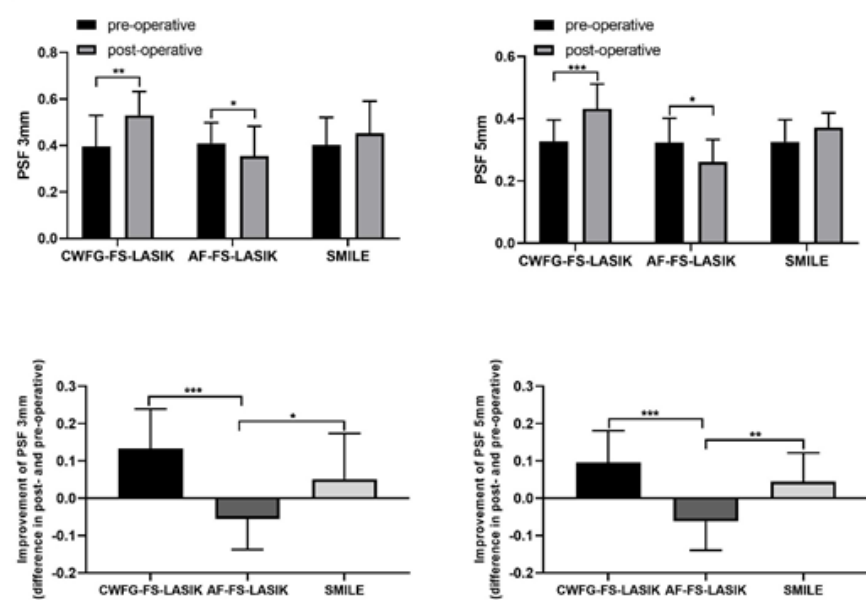

Figure 4

A Pre- and postoperative MTF and its improvement with the three surgical methods. B Pre- and postoperative PSF and its improvement with the three surgical methods. 